Article

\title{
Transdermal Optical Wireless Links with Multiple Receivers in the Presence of Skin-Induced Attenuation and Pointing Errors
}

\author{
George K. Varotsos ${ }^{1}\left(\mathbb{D}\right.$, Hector E. Nistazakis ${ }^{1, *}$, Konstantinos Aidinis ${ }^{2}$, F. Jaber ${ }^{3}{ }^{(D)}$ and \\ K.K. Mujeeb Rahman ${ }^{3}$ (D) \\ 1 Department of Electronics, Computers, Telecommunications and Control, Faculty of Physics, \\ National and Kapodistrian University of Athens, 15784 Athens, Greece \\ 2 Department of Electrical Engineering, Ajman University, Ajman P.O. Box 346, UAE \\ 3 Department of Biomedical Engineering, Ajman University, Ajman P.O. Box 346, UAE \\ * Correspondence: enistaz@phys.uoa.gr; Tel.: +30-210-7276710
}

Received: 22 May 2019; Accepted: 24 June 2019; Published: 28 June 2019

\begin{abstract}
The last few years, the scientific field of optical wireless communications (OWC) has witnessed tremendous progress, as reflected in the continuous emergence of new successful high data rate services and variable sophisticated applications. One such development of vital research importance and interest is the employment of high speed, robust, and energy-effective transdermal optical wireless (TOW) links for telemetry with implantable medical devices (IMDs) that also have made considerable progress lately for a variety of medical applications, mainly including neural recording and prostheses. However, the outage performance of such TOW links is significantly degraded due to the strong attenuation that affects the propagating information-bearing optical signal through the skin, along with random misalignments between transmitter and receiver terminals, commonly known as pointing error effect. In order to anticipate this, in this work we introduce a SIMO TOW reception diversity system that employs either OOK or more power-effective $L$-PPM schemes. Taking into account the joint impact of skin-induced attenuation and non-zero boresight pointing errors, modeled through the suitable Beckmann distribution, novel closed-form mathematical expressions for the average BER of the total TOW system are derived. Thus, the possibility of enhancing the TOW availability by using reception diversity configurations along with the appropriate modulation format is investigated. Finally, the corresponding numerical results are presented using the new derived theoretical outcomes.
\end{abstract}

Keywords: optical wireless communications (OWC); transdermal optical wireless (TOW) links; implantable medical devices (IMDs); skin-induced attenuation; non-zero boresight (NZB) pointing errors; single input-multiple output (SIMO) system; average bit error rate (ABER)

\section{Introduction}

Over the last few years, the growing increase in traffic carried by emerging telecommunication network applications, which require higher data rates, higher security levels, along with lower power consumption and cost, has triggered remarkable scientific interest in the transition from the increasingly crowed radio frequency (RF) spectrum to the more bandwidth-effective optical spectrum, where OWC, commonly known as Free Space Optical (FSO) communications, have attracted particular attention lately for a variety of applications in both medical and telecommunication fields [1,2]. In basic terms, this kind of technology uses an optical information-bearing carrier-i.e., visible, infrared, ultra-violet-which is propagated through the unguided transmission mediums such as atmosphere, 
space, seawater, or skin, in order to transmit the information signal from one end to another. Indeed, FSO links use either Lasers or LEDs (light emitting diodes) at the transmitter's side and photo-detectors at the receiver, in order to detect the propagating optical beam. In fact, by modulating the intensity and/or the phase of light waves traversing atmosphere, space, seawater, or even biological tissues, the FSO links can reliably transmit huge amounts of data. FSO systems have concrete advantages in comparison with their RF counterparts, mainly due to their operation at optical wavelengths. In fact, by increasing the carrier frequency from RF to that of optical waves, the information capacity is increased by many orders of magnitude. Thus, FSO can establish ultra-high bit rate wireless links. Furthermore, FSO systems can offer higher security level because of the very narrow laser beam that carries the information, which makes undetected interception difficult. Additionally, considering that FSO terminals are more compact and less heavy, FSO systems can be installed rapidly with low installation and operational cost. Moreover, they operate with lower power consumption in the unlicensed optical spectrum, while they are practically immune to multi-path dispersion and electromagnetic interference (EMI) [3-13].

By focusing on medical applications with IMDs that have made also notable advances lately in commercial and research field, FSO, in terms of TOW, is a prime candidate for effective high bandwidth transdermal communication, such as recording signals from in-body devices and actuating these devices from extra-body signals [14-17]. Nowadays, the common modalities used for transcutaneous communication are based on RF, acoustic wave, conductive, or electrical induction [9], and therefore, this kind of communication is realized by various conventional methods such as percutaneous wires, ultrasound or ultra-wide band (UWB) technology. Among them, percutaneous wires have a larger bandwidth and lower power consumption but physically traversing human skin increases the possibility of infection. Alternatively, an ultrasonic acoustic link has been used as a means of telemetry for cochlear implants, but utilizes piezoelectric materials that are not ideal for an IMD, due to high driving voltages [18]. On the other hand, wireless RF, which is a mature technology, is currently used in most implantable systems [19]. Indeed, RF telemetry has been used for diverse applications, including neuromuscular stimulators [17], commercial implantable cardioverter defibrillators and commercial cochlear implants for many years, while also neural microelectrode recording systems with integrated RF systems are being developed [20-22]. Current transdermal RF systems have been shown to achieve data rates up to several Mbps [20]. However, although a high-speed RF has been recently reported as being capable of transmitting data at $24 \mathrm{Mbps}$ [23], it still requires a large amount of power consumption, ca. $30 \mathrm{~mW}$. Additionally, transdermal RF systems cannot usually cover a sufficient bandwidth, since RF bandwidth is highly regulated. Indeed, the only available RF bands that could be used for high capacity wireless links are increasingly crowded, because of commonly used protocols and applications such as 802.11 , Bluetooth, and others. Moreover, RF transdermal links are highly vulnerable to EMI, and thus, susceptible to interference from other RF emitters and devices. It becomes now evident that exploiting the above-mentioned distinct advantages of FSO communication, TOW technology is a very promising alternative to all these conventional methods. Indeed, considering mainly the high data rates they provide (above $20 \mathrm{Mbps}$ rate which is the threshold to satisfy anticipated needs [24], especially with regard to neural recording [25-27]), their extremely high immunity to external interference along with their operation with low power consumption (of the $10 \mathrm{~mW}$ target [24]), TOW transmissions can meet easily the essential requirements of transdermal high-data-rate communication, including wireless operation, i.e., no wires traversing skin that could increase the possibility of infection and discomfort, high noise immunity to ensure operational independence of the ambient environment and operation with low-power consumption for the internal device to extend the longevity of the implant [14], which sometimes is strongly associated with patient's longevity.

However, the widespread deployment of TOW technology is significantly hampered by several performance-degrading factors that mainly arise from the variable characteristics and the nature of the skin as a transmission medium. Skin is a complex, multilayer, highly anisotropic biological 
entity-composed mainly of dermis, epidermis, and hypodermis—which entail many and different structural components such as cells, corpuscles, blood vessels, nerve endings, glands, muscles, and hair follicles. Consequently, light incident on skin is reflected, scattered, and absorbed by all its structural constituents that depend strongly on variable conditions and characteristics such as skin's thickness, tissues' heterogeneities, as well as different topology, region, race, gender, and race [28]. This leads to an important skin-induced loss in optical signal power, and hence, to a spatially broadened power distribution at the receiver, alike [29]. Specifically, photon absorption arise primarily from water content or hemoglobin and melanin, and can be minimized within the so-called skin's 'optical window', i.e., with radiation at wavelengths between $600 \mathrm{~nm}$ and $1300 \mathrm{~nm}$, while scattering, which is usually the most dominant process, happens mostly into the direction of photon travel, and thus, a highly forward-directed scattering occurs [30]. Due to the strong scattering induced by thick tissue, photons arrive in a very anisotropic pattern at the receiver and the power distribution is widely broadened, and as a result, a large photodiode should be used to collect the scattered photons [29]. However, larger size photodiodes not only lead to a limited bandwidth, and thus, to a lower link speed but also generate a greater input-referred noise [9,29,31]. Additionally, many commercially available emitters and photodiodes are optimized for operation in skin's 'optical window' range, as is the same wavelength range used for many fiber optics or free-air applications [32]. Nevertheless, this skin-induced attenuation described above remains a major concern in TOW communication since even for the highest tissue transmittance, i.e., for wavelengths between $800 \mathrm{~nm}$ and $1300 \mathrm{~nm}$, only $10 \%$ to $30 \%$ of incident optical power is transmitted through skin of typical thickness, i.e., $2 \mathrm{~mm}$ to $6 \mathrm{~mm}$, when the emitter and the detector are aligned [33-35]. Considering also the pointing errors due to unavoidable misalignments between transmitter and receiver terminals that is also an effect of primary concern in TOW communication, as well as in the wider FSO communication, the performance and availability of TOW transmissions is further degraded [2,4,7,36-39].

In view of the above improving communication with IMDs still remains a critical research issue due to the limitations including power loss, misalignment-induced fading, and the simultaneous need for very high data rates [40]. Thus, several works have been published by different research organizations around the world concerning optical transdermal transmission of data. In short, the feasibility of establishing TOW links has been experimentally verified in $[14,28,29,31]$. Specifically, in [28], it has been shown that FSO is an attractive solution in medical applications, in terms of TOW communication, because of its low power consumption that is of order of $\mu \mathrm{W}$ or below $10 \mathrm{mw}$ for very high data rates contrary to that of some tens of $\mathrm{mW}$ that their current RF counterparts require. Next, in [14] the concept of a "Medical Spectral Window", commonly known as skin's'optical window' mentioned above, has been experimentally proven. Within this wavelength region, optical radiation is least attenuated traversing human skin, and therefore, the propagating light experiences its maximum depth of penetration, penetrating into tissue as much as several centimeters [14,30,32,33,35]. Additionally, by using a vertical cavity surface emitting laser (VCSEL) with $850 \mathrm{~nm}$ wavelength very effective TOW experimental links have been reported in [19,29,35]. More precisely, in [35], data rates up to $16 \mathrm{Mbps}$ have been achieved through a skin thickness of $4 \mathrm{~mm}$ with a bit error rate (BER) of order of $10^{-9}$ and misalignment between transmitter and receiver terminals of $2 \mathrm{~mm}$, while consuming a power of $10 \mathrm{~mW}$. In [19], a TOW link capable of transmitting data at $50 \mathrm{Mbps}$ through sufficiently thick tissue, i.e., $2-6 \mathrm{~mm}$, with a BER below $1 \times 10^{-5}$ and power consumption as low as $1.1 \mathrm{~mW}$ to $6.4 \mathrm{~mW}$ respectively, considering $2 \mathrm{~mm}$ misalignment between terminals, while in [29] in vivo tests performed on an anesthetized sheep demonstrated transmitting data at $100 \mathrm{Mbps}$ with a BER below $2 \times 10^{-7}$, consuming only $2.1 \mathrm{~mW}$ of electrical power. With regard to pointing errors, in the majority of works concerning TOW communications, this effect has been either considered as a negligible one or it has been described by deterministic models, which are less suitable for the description of such random misalignment-induced irradiance fades [14,19,29,31].

Recently, in [36-38], the authors considered the stochastic nature of a TOW link and derived closed-form expressions for the average signal-to-noise-ratio (SNR) and its outage performance, 
in terms of outage probability metric. In basic terms, they demonstrated that pointing errors can degrade the TOW performance by more than $10 \%$. However, in the latter works, the stochastic nature of misalignment due to the relative motion between the transmitter and the receiver terminals is modeled by the commonly used model that has been proposed in [7] for outdoor FSO communication links, which takes into account the jitter but ignores the non-zero boresight displacement. Indeed, pointing errors consist of two components, i.e., boresight and jitter. The former is the fixed displacement between the beam and the detector center, while the latter is the random offset of the beam center at detector plane [41]. Thus, we should use a more generalized and practical pointing error model, commonly known as nonzero boresight (NZB) pointing error model, where apart from the effect of beam width, detector size, and different jitters for the elevation and horizontal displacement, the effects of NZB errors are also taken into consideration [42]. For the sake of clarity, this boresight displacement must be necessarily always considered at the receiver side when more than one detector aperture is employed, since each laser can be strictly aligned with only one receive aperture. Hence, there is an inherent boresight displacement that depends on the spacing among receiver detectors, due to the different distances from the specific detector aperture that determines the alignment point [41].

Nevertheless, NZB pointing errors had never been examined in TOW literature, just as no method had been proposed in order to enhance the TOW outage performance and availability. Only, very recently, authors demonstrated for the first time, in [39], that a diversity method-i.e., in space, in time, or in wavelength-is an effective technique to enhance the outage performance of a typical TOW in terms of outage probability metric, by considering the joint impact of both attenuation-induced fading and misalignment-induced fading with NZB, modeled by the accurate Beckmann distribution [3,41-46]. In basic terms, diversity refers to the consideration of multiple copies of the propagated signals in an attempt to overcome a poor transmission media state so as to reinforce the communication link's availability and robustness. Diversity can be realized either in space, in time or in wavelength [3,4,47-51]. Considering spatial diversity $[47,50]$, an FSO system employs multiple transmitters and/or receivers at different places that transmit and receive copies from the same information-bearing signal, resulting in an enhanced error performance. Through time diversity $[49,52,53]$, an FSO system employs a single transmitter-receiver pair, but the signal is retransmitted at different time slots. Finally, assuming wavelength diversity [49,54], an FSO system uses a composite transmitter, and thus, copies of the same signal are transmitted at the same time at different wavelengths towards a number of receivers [51].

It becomes clear that via diversity an initial single input-single output (SISO) FSO link is transformed into a more performance effective, by means of error rate and availability, single input-multiple output (SIMO), multiple input-single output (MISO), or multiple input-multiple output (MIMO) FSO system. Additionally, to the best of the authors' knowledge, in previous works related to TOW communication, only the simplest, but not always efficient enough, intensity modulation/direct detection (IM/DD) with on-off keying (OOK) modulation formats have been investigated. Although OOK is the most widely used modulation scheme in FSO area mainly due to its simplicity, when it is operating under fading conditions the detector threshold must be estimated and adopted by appropriate means, according to the variable channel states. This drawback can be circumvented via Pulse Position Modulation (PPM), which outperforms OOK in power efficiency [3,55-57]. The latter happens at the expense of higher complexity and lower spectral efficiency, which is however, of minor importance considering the huge, in any case, available optical modulation bandwidth. Moreover, to the best of our knowledge, TOW links have been evaluated only in terms of instantaneous BER, when the reality is that the average bit error rate (ABER) metric is recommended in order to be able to draw meaningful conclusions for their reliability.

Motivated by these facts, a key novelty in this work is the estimation of the ABER performance of a SIMO TOW system that may employ apart from OOK, L-PPM modulation format alike, under the joint impact of skin-induced attenuation and pointing errors with non-zero boresight. It should be highlighted here that in previous published works concerning FSO, receiver's diversity in terms of 
SIMO configurations have been used in order to enhance the performance of a terrestrial, outdoor FSO communication link, and thus, random atmospheric channel's impairments have been addressed, such as atmospheric turbulence-induced signal's fading [2,4,46], while in [2] L-PPM signaling technique has been proposed in order to achieve further such performance enhancements. However, for a TOW link which is investigated in this work, there is an essential difference concerning the nature of the transmission medium. In this work, the transmission medium is biological tissue instead of atmosphere. In fact, the examined channel is the skin which is totally different and more complex for the optical signal in comparison with atmosphere. Thus, apart from non-zero boresight pointing errors which arise from random movements between transmitter and receiver terminals, skin-induced attenuation that experiences the information-bearing optical signal is now considered, while atmospheric turbulence is obviously neglected. In short, although SIMO and L-PPM techniques have been also considered in outdoor FSO communications, this is the first time that both techniques are applied this way in the biomedical and transdermal communication systems where the requirements for very low energy consumption and negligible biological effects must be fully met. Thus, motivated and inspired by our previous works in outdoor FSO $[2,4,46]$, in the current work we introduce, for the first time ever, SIMO with L-PPM in an entirely different field of optical wireless communications-i.e., transdermal optical communications with medical implants-as an attempt to enhance the outage probability of such systems. Thus, the scope of this work is to outbalance the negative side-effects of this joint impact by introducing L-PPM SIMO TOW configurations. Therefore, an ABER analysis is presented and novel closed-form mathematical expressions are derived for its estimation. In this context, a performance comparison in terms of ABER between SISO and SIMO TOW configurations with either OOK or L-PPM formats is performed, as an attempt to reveal possible significant ABER performance enhancements and/ or IMD's lower power consumption achievements that stem from the selection of proper SIMO L-PPM TOW system configurations.

\section{System Model}

\subsection{Signal Model}

The TOW system under consideration consists of three fundamental components, i.e., the out-of-body unit, the skin channel and the $M$ in-body unit(s). In this respect, the system employs $M$ direct TOW links, where the $M$ optical signal's copies pass through skin channel once. The whole link appears in Figure 1.

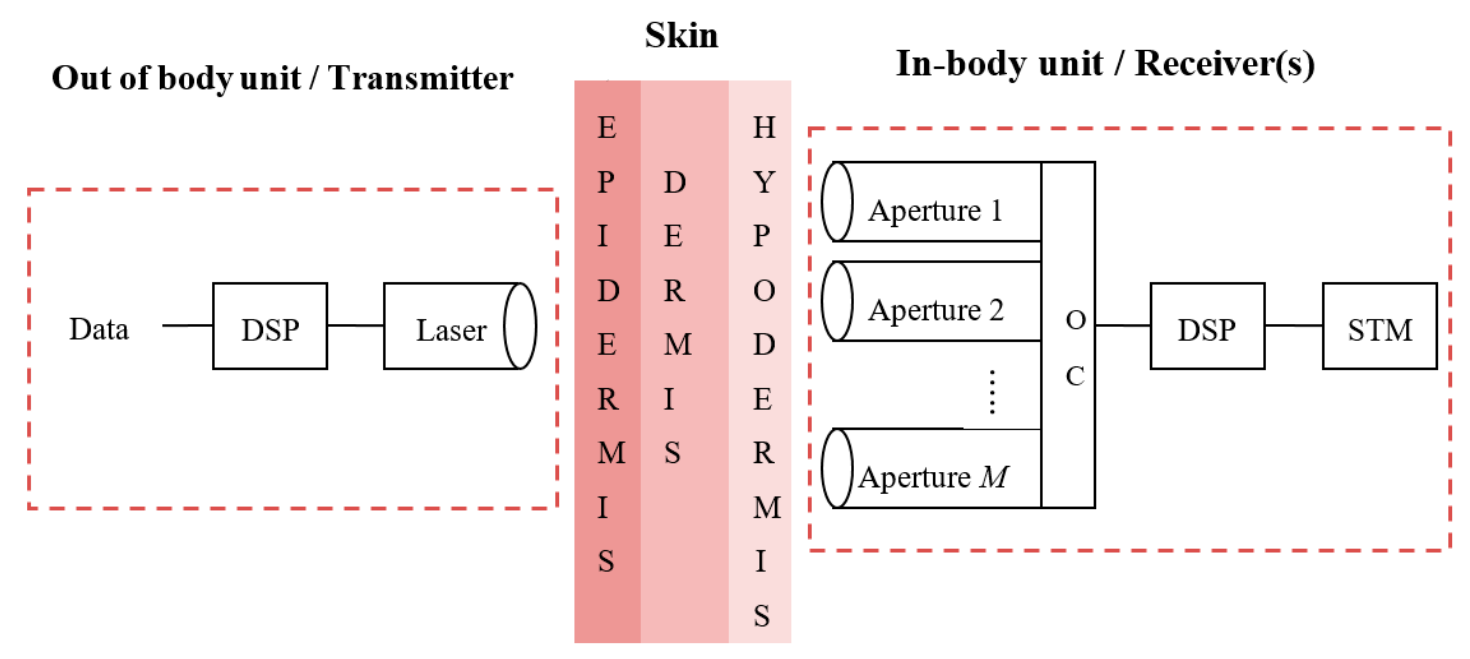

Figure 1. Block diagram of the proposed TOW system.

It is assumed that that the external unit consists of a data capturing unit, followed by a digital signal processing (DSP) unit. The former unit converts external stimulations into electrical signals, 
while the latter unit performs the processes of digitization and compression of the $M$ data copies into $\mathrm{OOK}$, IM/DD, or L-PPM modulated signals. These signals are forwarded to the transmitter, which emits $M$ data copies of the same part of information towards the internal unit that consists, in turn, of the corresponding receiver(s), DSP unit and the stimulation (STM) unit that eventually stimulates the appropriate nerve. Thus, the internal DSP and STM units are responsible for generating the appropriate stimulations [14,36-39]. In this context, the statistical channel model for the examined TOW system is given as [39]

$$
y_{m}=\eta_{m} h_{m} x+n,
$$

where $y_{m}$ represents the $m$-th signal copy with $m=1,2, \ldots, M, \eta_{m}$ is the effective receiver's photo-current conversion ratio, $x$ is the modulated signal, convey over the corresponding $h_{m}$ channel with additive noise $n$ that is modeled as a zero-mean complex Gaussian process with variance $\sigma^{2}$.

The $m$-th channel for the case of the transdermal link can be expressed as [36-39,58]

$$
h_{m}=h_{l, m} h_{p, m}
$$

where $h_{l, m}$ stands for the deterministic channel coefficient due to the propagation losses of the $m$-th channel though the skin and $h_{p, m}$ represents the corresponding stochastic process that models the geometric spread due to the presence of pointing errors. The former deterministic term is expressed as [36-39]

$$
h_{l, m}=\exp \left[-\frac{1}{2} \alpha\left(\lambda_{m}\right) \delta_{m}\right]
$$

where $\delta_{m}$ is the total dermis thickness of the $m$-th channel and $\alpha\left(\lambda_{m}\right)$ is the corresponding skin attenuation coefficient in the corresponding operational wavelength $\lambda_{\mathrm{m}}$. More specifically, the term skin refers to the complex biological structure that consists of three basic layers, i.e., stratum corneum, epidermis and dermis, with transmitter and receiver terminals being in contact with the epidermal and the adipose side of skin [28]. Thus, their distance can be accurately approximated by skin's thickness while the parameter $h_{l, m}$ expresses the skin-induced attenuation of the propagated optical signal through the $m$-th transdermal path. The wavelength dependent value of $\alpha\left(\lambda_{m}\right)$, depends also on the optical properties of the skin and for values between $400 \mathrm{~nm}$ and $1800 \mathrm{~nm}$ is obtained as [37-39]

$$
\alpha\left(\lambda_{m}\right)=\sum_{i=1}^{8} a_{i} \exp \left[-\left(\frac{\lambda_{m}-b_{i}}{c_{i}}\right)^{2}\right],
$$

where $\lambda_{m}$ values are expressed in $\mathrm{nm}$ and the values of $a_{i}, b_{i}$ and $c_{i}$, with $i=1,2 \ldots, 8$, are given in the following table as $[37,38]$.

\subsection{Generalized Pointing Errors Model with Non-Zero Boresight}

Principally, pointing errors include the random beam offset and the fixed displacement between the beam and detector-i.e., the jitter and the boresight component-respectively [41]. Hence, when a Gaussian beam propagates through distance $\delta_{m}$ from the transmitter to a circular detector with aperture radius $r_{m}$, the fraction of the collected power at receiver can be approximated as [42]

$$
h_{p}\left(R_{m}, \delta_{m}\right) \approx A_{0, m} \exp \left(-\frac{2 R_{m}^{2}}{w_{e q, m}^{2}}\right), \quad R_{m} \geq 0,
$$

where $w_{\text {eq, } m}$ represents the equivalent beam radius in the $m$-th receiver aperture which is given as $w_{e q, m}=\left[\sqrt{\pi} \operatorname{erf}\left(v_{m}\right) w_{\delta, m}^{2} / 2 v_{m} \exp \left(-v_{m}^{2}\right)\right]^{1 / 2}$, with $v_{m}=\sqrt{\pi} r_{m} / \sqrt{2} w_{\delta, m}$ and $\operatorname{erf}($.$) denotes the error$ function [59], (8.250.1). Additionally, in TOW links the parameter $w_{\delta, m}=\delta_{m} \tan \left(\theta_{m} / 2\right)$, stands for the $m$-th beam waist on the receiver plane at distance $\delta_{m}$ from the transmitter [36-39], with $\theta_{m}$ being the corresponding divergence angle. Furthermore, $A_{0, m}=\operatorname{erf}^{2}\left(v_{m}\right)$ is the fraction of the collected 
power at $r_{m}=0[7,42]$. Moreover, $R_{m}$ is the radial displacement at the $m$-th receiver that is expressed as $R_{m}=\left|\vec{R}_{m}\right|=\sqrt{R_{x, m}^{2}+R_{y, m}^{2}}$ where $\vec{R}_{m}=\left[R_{x, m}, R_{y, m}\right]^{T}$ is the radial displacement vector with $R_{x, m}$, $R_{y, m}$, representing the displacements located along the horizontal and elevation axes at the detector plane, respectively. These variables are considered as nonzero mean Gaussian distributed random variables, i.e., $R_{x, m} \sim N\left(\mu_{x, m}, \sigma_{x, m}^{2}\right), R_{y, m} \sim N\left(\mu_{y, m}, \sigma_{y, m}^{2}\right)$ where $\mu_{x, m}, \mu_{y, m}$, denote their mean values and $\sigma_{x, m}, \sigma_{y, m}$, the jitters for horizontal and elevation displacements, respectively [42]. Here, it should be clearly outlined that the approximation in (5) is accurate enough for $w_{\delta, m} / r_{m}>6$, which is satisfied in typical FSO systems.

Hence, a generalized statistical model which can accurately emulate the pointing errors effect considering the beam width, the detector size, the different jitter for the elevation and the horizontal displacements and the NZB error, is Beckmann's model with the following probability density function (PDF) [58],

$$
\begin{aligned}
f_{R, m}\left(R_{m}\right) & =\frac{R_{m}}{2 \pi \sigma_{x, m} \sigma_{y, m}} \\
& \times \int_{0}^{2 \pi} \exp \left[-\frac{\left(R_{m} \cos \varphi_{m}-\mu_{x, m}\right)^{2}}{2 \sigma_{x, m}^{2}}-\frac{\left(R \sin \varphi_{m}-\mu_{y}\right)^{2}}{2 \sigma_{y}^{2}}\right] d \varphi_{m},
\end{aligned}
$$

where $\varphi_{m}$ stands for the transmit divergence angle, which denotes the increase in beam radius with distance from the transmitter [42].

According to the analysis performed in [42], the Beckmann's distribution in (6) can be accurately approximated and simplified through the following modified Rayleigh distribution [42]

$$
f_{R, m}\left(R_{m}\right)=\frac{R_{m}}{\sigma_{\mathrm{mod}, m}^{2}} \exp \left(-\frac{R_{m}^{2}}{2 \sigma_{\mathrm{mod}, m}^{2}}\right), \quad R_{m} \geq 0,
$$

where the modified jitter is expressed as $[3,46]$

$$
\sigma_{\text {mod }, m}^{2}=\left(\frac{3 \mu_{x, m}^{2} \sigma_{x, m}^{4}+3 \mu_{y, m}^{2} \sigma_{y, m}^{4}+\sigma_{x, m}^{6}+\sigma_{y, m}^{6}}{2}\right)^{1 / 3} .
$$

Consequently, the PDF for $h_{p, m}$ is approximated as $[3,46]$

$$
f_{h_{p, m}}\left(h_{p, m}\right)=\frac{\psi_{m}^{2} g_{m}^{-\psi_{m}^{2}}}{A_{0, m}^{\psi_{m}^{2}}} h_{p, m}^{\psi_{m}^{2}-1}, 0 \leq h_{p, m} \leq g_{m} A_{0, m},
$$

where in each link it holds that $\psi_{m}=w_{e q, m} / 2 \sigma_{\bmod , m}$, with $\psi_{x, m}=w_{e q, m} / 2 \sigma_{x, m}, \psi_{y, m}=w_{e q, m} / 2 \sigma_{y, m}$ and $g_{m}=\exp \left(\frac{1}{\psi_{m}^{2}}-\frac{1}{2 \psi_{x, m}^{2}}-\frac{1}{2 \psi_{y, m}^{2}}-\frac{\mu_{x, m}^{2}}{2 \sigma_{x, m}^{2} \psi_{x, m}^{2}}-\frac{\mu_{y, m}^{2}}{2 \sigma_{y, m}^{2} \psi_{y, m}^{2}}\right)[39,41,46,58]$. Here, it becomes evident that the impact of pointing errors effect is getting stronger as the value of $\psi_{m}$ parameter decreases. Conversely, for very large values of $\psi_{m}$, pointing errors effect can be considered as a negligible one. Moreover, considering independent identical zero mean Gaussian distributions for both elevation and horizontal displacement with $g_{m}=1, \quad \mu_{x, m}^{2}=0, \quad \mu_{y, m}^{2}=0, \quad \sigma_{x, m}^{2}=\sigma_{y, m}^{2}=\sigma_{s}^{2}$, the generalized PDF for pointing errors with NZB in (9), can be simplified to the one for zero boresight misalignments presented in [7].

\subsection{Joint Impact of Skin-Induced Attenuation and NZB Pointing Errors}

Once we obtained the expressions for skin-induced attenuation through the parameter $h_{l, m}$ in (3) and misalignment induced fading with NZB the through the parameter $h_{p, m}$ in (9) respectively, we can now estimate their combined impact through their product in (2), i.e., through the parameter 
$h_{m}$. Indeed, since the parameter $h_{l, m}$ is a deterministic one, by both using (2), (3), and (9) and applying a random variable (RV) transformation [60], the joint PDF of the random variable $h_{m}$ is obtained as

$$
f_{h_{m}}\left(h_{m}\right)=\frac{\psi_{m}^{2}}{\left(A_{0, m} g_{m}\right)^{\psi_{m}^{2}}} h_{l, m}^{\psi_{m}^{2}-1} h_{m}^{-\psi_{m}^{2}} .
$$

Additionally, based on (1)-(3) the instantaneous electrical SNR, $\gamma$, is given as [39]

$$
\gamma_{m}=\frac{\eta_{m}^{2} \exp \left(-\alpha\left(\lambda_{m}\right) \delta_{m}\right) h_{p, m}^{2} \widetilde{P}_{S}}{N_{0}}
$$

where $\widetilde{P}_{S}$ and $N_{0}$ rerpresent the signal and noise optical power spectral density (PSD), respectively.

Since $\mathrm{E}\left[h_{m}\right]=\int_{0}^{\infty} h_{m} f_{h_{m}}\left(h_{m}\right) d h_{m}$, by using (10), the average electrical SNR in the case of TOW links with NZB errors is obtained as [37]

$$
\mu_{m}=\frac{\eta_{m}^{2} \mathrm{E}^{2}\left[h_{m}\right] \widetilde{P}_{S}}{N_{0}}=\frac{\eta_{m}^{2} \psi_{m}^{2} A_{0, m}^{2} g_{m}^{2} \exp \left(-\alpha\left(\lambda_{m}\right) \delta_{m}\right) \widetilde{P}_{S}}{\left(\psi_{m}^{2}+2\right) N_{0}},
$$

while for the zero boresight scenario, the expected electrical SNR, $\mu_{m, z e r o}$, is given by setting in (12) that $g_{m}=1$, while also it holds that $\mu_{x, m}^{2}=0, \quad \mu_{y, m}^{2}=0, \quad \sigma_{x, m}^{2}=\sigma_{y, m}^{2}=\sigma_{s}^{2}$.

More precisely, for SIMO spatial diversity FSO systems the nonzero-boresight electrical SNR could be considered as equal to the zero-boresight scenario only for the simplified SISO configuration, where $M=1$, i.e., $\mu_{1}=\mu_{1, z e r o}=\mu$. This denotes that the positioning of the laser is assumed to be performed in regard to the corresponding photodetector and thus only zero-boresight pointing errors are addressed. On the contrary, when $M>1$, the boresight is equal zero only to one branch, while the others should also deal with nonzero boresight components. Indeed, the laser cannot be simultaneously strictly aligned with all the additional photodiodes as effectively as with the initial one. Hence, NZB pointing errors are emerged for the rest receiver apertures, where lower amount of optical power arrives at their side. Thus, the nonzero boresight consideration through (12) brings about lower electrical SNR values, i.e., $\mu_{1}<\mu_{1, \text { zero }}$ for $1<m \leq M$ [2]. Consequently, it becomes evident from the above that by estimating the expected value of $h_{m}$, we conclude that the average electrical SNR in the case of TOW links depends strongly on pointing errors effect with or without non-zero boresight components. As we will more clearly see below, this is a very important outcome for the outage performance estimation as a function of the average SNR for a TOW link with pointing errors.

\section{ABER Analysis of the SISO TOW Link}

The BER is a critical metric for the availability and performance estimation of each communication system, such as TOW links. Nevertheless, the BER is estimated through the instantaneous electrical SNR at the receiver, $\gamma_{m}$, which experiences random fluctuations, and thus, the BER metric obtains random values as well. Thus, in order to draw more accurate conclusions for the system performance in practice we need to estimate the ABER metric. Consequently, in this section we first investigate the ABER for an IM/DD SISO TOW link with OOK, and then, we expand this work for an IM/DD SIMO TOW system with OOK and L-PPM formats with optimal combining (OC) [2,51].

\subsection{SISO TOW Link with IM/DD OOK}

The OOK scheme is the most widely used modulation format for FSO communication systems, mainly due to its simplicity, ease in installation, simple receiver design, bandwidth efficiency, low cost, and immunity to the innate nonlinear ties of the laser and the external modulator $[57,61]$. However, when OOK is employed through a transmission medium of varying characteristics, in order to 
optimally operate, the knowledge of an adaptive threshold for each specific channel state is necessarily required [2].

Assuming an IM/DD TOW SISO link with OOK, errors may be occurred at the receiver in determining the actual symbols transmitted, because of the presence of AWGN [2]. Thus, the instantaneous BER is estimated through the following expression $[47,50,52]$

$$
P_{e}=p(0) P(e \mid 0)+p(1) P(e \mid 1),
$$

where $p(0)$ and $p(1)$ are the probabilities of transmitting the bits " 0 " and " 1 " respectively, while $P(e \mid 0)$ and $P(e \mid 1)$ are their corresponding conditional bit-error probabilities [47,62]. Without loss of generality, assuming that $p(0)=p(1)=1 / 2$ and $P(e \mid 0)=P(e \mid 1)$, and assuming $m=1$, because SISO link is considered, and $\widetilde{P}_{S}=1 \mathrm{mw} / \mathrm{MHz}$, the BER is given as $[47,50,52]$

$$
P_{e}(h)=P(e \mid 0, h)=P(e \mid 1, h)=P\left(n>\frac{\eta h}{2}\right)=P\left(n<-\frac{\eta h}{2}\right)=\frac{1}{2} \operatorname{erfc}\left(\frac{\eta h}{2 \sqrt{N_{0}}}\right),
$$

where $\operatorname{erfc}($.$) stands for the complementary error function [59], (8.250.4). By averaging (14) over h$, the ABER, $P_{e, a v}$, is obtained as $[47,52]$

$$
P_{e, a v}=\int_{0}^{\infty} P_{e}(h) f_{h}(h) d h
$$

Thus, by substituting (10) into (15) we get

$$
P_{e, a v}^{O O K}=\frac{1}{2} \frac{\psi^{2} h_{l}^{\psi^{2}-1}}{\left(A_{0} g\right)^{\psi^{2}}} \int_{0}^{\infty} \operatorname{erfc}\left(\frac{\eta h}{2 \sqrt{N_{0}}}\right) h^{-\psi^{2}} d h,
$$

and by using then $\int_{0}^{\infty} t^{z-1} \operatorname{erf} c(t) d t=\frac{1}{\sqrt{\pi} z} \Gamma\left(\frac{z+1}{2}\right), \operatorname{Re}(z)>0$ [63], (06.2721.0132.01), we get after some calculations the following ABER expression for the IM/DD OOK TOW link, by taking into account the skin-induced attenuation and NZB pointing errors

$$
P_{e, a v}^{O O K}=\frac{\psi^{2} \exp \left(-\frac{1}{2} \alpha(\lambda) \delta\right)^{\psi^{2}-1}}{2 \sqrt{\pi}\left(1-\psi^{2}\right)\left(A_{0} g\right)^{\psi^{2}}}\left(\frac{\eta}{2 \sqrt{N_{0}}}\right)^{\psi^{2}-1} \Gamma\left(\frac{2-\psi^{2}}{2}\right) .
$$

Furthermore, by substituting (12) into (17) we can alternatively express the ABER as a function of the average electrical SNR, $\mu$

$$
P_{e, a v}^{O O K}=\frac{\psi^{2}\left[\mu\left(\psi^{2}+2\right)\right]^{\frac{\psi^{2}-1}{2}} \Gamma\left(\frac{2-\psi^{2}}{2}\right)}{2^{2} \sqrt{\pi} \psi^{2}-1\left(1-\psi^{2}\right)\left(A_{0} g\right)^{2 \psi^{2}-1}\left(\widetilde{P}_{s}\right)^{\frac{\psi^{2}-1}{2}}} .
$$

\subsection{SISO TOW Link with IM/DD L-PPM}

The PPM modulation format is more power effective than OOK, but at the expense of an increased bandwidth requirement and higher complexity. Additionally, PPM eliminates the requirement of OOK for a decision threshold dependent on the input power [2,57]. In $L$-PPM, each word of $N$ bits is mapped into one of the $L=2^{N}$ symbols and transmitted through the channel, while each $L$-PPM symbol has the form of a pulse transmitted in one of the $L=2^{N}$ consecutive time slots with duration $T_{S}=N T_{b} / L$, where $T_{b}$ is the bit duration, with the remaining slots being empty. Information is encoded within the position of a pulse of constant power along with $(\mathrm{N}-1)$ empty slots, while the position of the pulse 
corresponds to the decimal values of the $N$-bit input data [64]. Assuming a SISO L-PPM TOW link we can omit, for the sake of brevity, the index $m$, and thus, the BER as a function of $h$, can be defined as [56]

$$
P_{e}^{L-P P M}(h)=\frac{1}{2} \operatorname{erfc}\left(\sqrt{\frac{\eta^{2} h^{2}}{8 N_{0}} L \log _{2} L}\right) .
$$

Next, in order to estimate the ABER of the TOW link with the L-PPM scheme under the joint impact of skin-induced attenuation and NZB pointing errors, we average (19) over $h$. After substituting (10) and (19) into (15), we get

$$
P_{e, a v}^{L-P P M}=\frac{\psi^{2} \exp \left(-\frac{1}{2} \alpha(\lambda) \delta\right)^{\psi^{2}-1}}{2 \sqrt{\pi}\left(1-\psi^{2}\right)\left(A_{0} g\right)^{\psi^{2}}}\left(\frac{\eta \sqrt{L \log _{2} L}}{\sqrt{8 N_{0}}}\right)^{\psi^{2}-1} \Gamma\left(\frac{2-\psi^{2}}{2}\right) .
$$

Alternatively, by substituting (12) into (20), the latter can be expressed as a function of the average electrical SNR, $\mu$

$$
P_{e, a v}^{L-P P M}=\frac{\psi^{2}\left[\mu\left(\psi^{2}+2\right)\right]^{\frac{\psi^{2}-1}{2}}\left(\frac{\sqrt{L \log _{2} L}}{8}\right)^{\frac{\psi^{2}-1}{2}} \Gamma\left(\frac{2-\psi^{2}}{2}\right)}{2 \sqrt{\pi} \psi^{2}-1\left(1-\psi^{2}\right)\left(A_{0} g\right)^{\psi^{2}}\left(\widetilde{P}_{s}\right)^{\frac{\psi^{2}-1}{2}}} .
$$

\section{ABER for SIMO TOW Systems with Receivers' Diversity}

In this Section, we investigate spatial, time or wavelength diversity implementations, in terms of a SIMO optical communication system. In this context, it is assumed that the transmitter emits $M$ copies of the same part of the information signal towards $M$ receivers for the case of spatial diversity configuration or in $M$ different time moments at the same receiver, for the time diversity case [4]. However, in time diversity system, since the transmitter is emitting $M$ copies of the same signal in $M$ different moments and the channel characteristics randomly change over time, each propagating copy addresses different channel characteristics. Consequently, $M$ different virtual propagation paths (or receivers) can be considered and thus, this time diversity system can be also emulated as a SIMO one. In reality, each copy arrives at the receiver and remains at the buffer of the system until all the $M$ copies been received. Thus, each copy of the information signal propagates through the same path but with different channel's characteristics, while the total information signal at the receiver buffer consists of $M$ different versions of the same initial signal [4,52]. Finally, in wavelength diversity a composite transmitter simultaneously emits $M$ copies of the same part of the information signal at $M$ different optical wavelengths towards $M$ corresponding receivers [51].

\subsection{SIMO TOW System with IM/DD OOK}

The optimum decision metric for a SIMO TOW link with IM/DD OOK is obtained as $[47,50]$

$$
P\left(\vec{y} \mid \text { on }, h_{m}\right) \underset{\text { off }}{\stackrel{\text { on }}{>}} P\left(\vec{y} \mid o f f, h_{m}\right),
$$

where $\vec{y}=\left[y_{1}, y_{2}, \ldots, y_{M}\right]$ is the vector signal that describes the corresponding emissions $m=1,2, \ldots M$, in different receivers or in different time slots the $M$ copies of the signal. Recall that considering OOK the condition 'on' refers to presence of light at the receiver's side, i.e., to the transmission of bit ' 1 ', while the condition 'off' corresponds to light's absence, i.e., to the transmission of bit ' 0 ', respectively. Additionally, one of the most significant schemes for the signal reception is the OC $[47,50,52]$. Considering OC diversity with $M$ branches at the receiver, aperture area of each 
detector is $M$ times smaller than the area of detector when there is no diversity implementation. Thus, as a consequence of the background radiation noise, the variance of the noise in each detector at receiver is $M$ times smaller than the noise variance of the system without diversity implementation, i.e., $\sigma_{m, n}^{2}=\sigma_{n}^{2} / M=N_{0} /(2 M)$. Here, the ABER is given as [50]

$$
P_{e, a v, M}^{O O K}=\int_{\mathrm{h}} f_{\mathrm{h}}(\mathrm{h}) Q\left[\frac{1}{\sqrt{2 M N_{0}}} \sqrt{\sum_{m=1}^{M}\left(\eta_{m} h_{m}\right)^{2}}\right] d \mathrm{~h},
$$

where $\mathrm{h}=\left[h_{1}, h_{2}, \ldots, h_{M}\right]$ is the vector of the TOW channel state for each one the $M$ copies of the signal. In order to estimate the multiple integral in (23) we initially substitute the approximation of [65] for the Q-function, $Q(x) \approx \frac{e^{-x^{2} / 2}+3 e^{-2 x^{2} / 3}}{12}$, into (23). Note that it practically holds that $0.5 \leq \eta_{m} \leq 0.9$. Additionally, the parameter $h_{\mathrm{m}}$ obtains non-negative values, i.e., $0 \leq h_{m} \leq 1$, with $h=h_{l} h_{p}$. Specifically considering that for the optical medical window $700 \mathrm{~nm} \leq \lambda_{m} \leq 1300 \mathrm{~nm}$ and that $2 \mathrm{~mm} \leq \delta_{m} \leq 8 \mathrm{~mm}$ for typical TOW links, by using (3), (4), and Table 1 we get that $0.0003 \leq h_{l, m} \leq 0.23$, while also from (9), $0 \leq h_{p, m} \leq g_{m} A_{0, m}$, i.e., $0 \leq h_{p, m} \leq 1$. Moreover, it practically holds that $1 \leq M \leq 5$ and that $N_{0}=(1.3 p A / \sqrt{H z})^{2}$. Under these parameter values, this approximation for the Q-function is valid. Therefore, the obtained multiple integral can be estimated through the following multiplication of single integrals [66]

$$
P_{e, a v, M}^{O O K} \approx \frac{1}{12} \prod_{m=1}^{M} \int_{0}^{\infty} \exp \left(-\frac{\eta_{m}^{2} h_{m}^{2}}{4 M N_{0}}\right) f_{h_{m}}\left(h_{m}\right) d h_{m}+\frac{1}{4} \prod_{m=1}^{M} \int_{0}^{\infty} \exp \left(-\frac{\eta_{m}^{2} h_{m}^{2}}{3 M N_{0}}\right) f_{h_{m}}\left(h_{m}\right) d h_{m} .
$$

Next, by substituting (10) into (24) we get

$$
\begin{aligned}
P_{e, a v, M}^{O O K} & \approx \frac{1}{12} \frac{h_{l, m}^{\psi_{m}^{2}-1} \psi_{m}^{2}}{\left(A_{0, m} g_{m}\right)^{\psi_{m}^{2}}} \prod_{m=1}^{M} \int_{0}^{\infty} \exp \left(-\frac{\eta_{m}^{2} h_{m}^{2}}{4 M N_{0}}\right) h_{m}^{-\psi_{m}^{2}} d h_{m} \\
& +\frac{1}{4} \frac{h_{l, m}^{\psi^{2}-1} \psi_{m}^{2}}{\left(A_{0, m} g_{m}\right)^{\psi_{m}^{2}}} \prod_{m=1}^{M} \int_{0}^{\infty} \exp \left(-\frac{\eta_{m}^{2} h_{m}^{2}}{3 M N_{0}}\right) h_{m}^{-\psi_{m}^{2}}\left(h_{m}\right) d h_{m}
\end{aligned}
$$

Thus, by using $\int_{0}^{\infty} x^{n} e^{-z x^{2}} d x=\frac{\Gamma[(n+1) / 2]}{2 z^{\frac{n+1}{2}}}, n>-1, z>0$ [59], and by substituting (3) in (25), the ABER of a IM/DD OOK SIMO TOW system with OC under both skin-induced attenuation and NZB pointing errors conditions, is estimated as

$$
\begin{aligned}
P_{e, a v, M}^{\mathrm{OOK}} \approx & \approx \frac{1}{12} \prod_{m=1}^{M} \frac{\psi_{m}^{2} \exp \left(-\alpha\left(\lambda_{m}\right) \delta_{m} / 2\right)^{\psi_{m}^{2}-1} \Gamma\left[\left(1-\psi_{m}^{2}\right) / 2\right]}{2\left(\eta_{m}^{2} / 4 M N_{0}\right)^{\frac{1-\psi_{m}^{2}}{2}}\left(A_{0, m} g_{m}\right)^{2}} \\
& +\frac{1}{4} \prod_{m=1}^{M} \frac{\psi_{m}^{2} \exp \left(-\alpha\left(\lambda_{m}\right) \delta_{m} / 2\right)^{\psi_{m}^{2}-1} \Gamma\left[\left(1-\psi_{m}^{2}\right) / 2\right]}{2\left(\eta_{m}^{2} / 3 M N_{0}\right)^{\frac{1-\psi_{m}^{2}}{2}}\left(A_{0, m} g_{m}\right)^{\psi_{m}^{2}}}
\end{aligned}
$$

Alternatively, by substituting (12) into (26) we get the latter as a function of the average electrical SNR, $\mu_{m}$

$$
\begin{aligned}
P_{e, a v, M}^{\mathrm{OOK}} \approx & \approx \frac{1}{12} \prod_{m=1}^{M} \frac{\psi_{m}^{3-\psi_{m}^{2}}\left(A_{0, m} g_{m}\right)^{1-2 \psi_{m}^{2}}\left(4 \widetilde{P}_{S} M\right)^{\frac{1-\psi_{m}^{2}}{2}} \Gamma\left[\left(1-\psi_{m}^{2}\right) / 2\right]}{2\left[\mu_{m}\left(\psi_{m}^{2}+2\right)\right]^{\frac{1-\psi_{m}^{2}}{2}}} \\
& +\frac{1}{4} \prod_{m=1}^{M} \frac{\psi_{m}^{3-\psi_{m}^{2}}\left(A_{0, m} g_{m}\right)^{1-2 \psi_{m}^{2}}\left(3 \widetilde{P}_{S} M\right)^{\frac{1-\psi_{m}^{2}}{2}} \Gamma\left[\left(1-\psi_{m}^{2}\right) / 2\right]}{2\left[\mu_{m}\left(\psi_{m}^{2}+2\right)\right]^{\frac{1-\psi_{m}^{2}}{2}}}
\end{aligned}
$$


Table 1. Parameter $a_{i}, b_{i}$ and $c_{i}$ values.

\begin{tabular}{cccc}
\hline $\mathbf{i}$ & $\mathbf{a}_{\mathbf{i}}$ & $\mathbf{b}_{\mathbf{i}}$ & $\mathbf{c}_{\mathbf{i}}$ \\
\hline 1 & 10 & 0.35 & 0.065 \\
2 & 4.5 & 0.42 & 0.25 \\
3 & 13.48 & -1.5 & 50.12 \\
4 & 14.7 & 1442 & 49.35 \\
5 & 7.435 & 1499 & 75.88 \\
6 & 48 & 3322 & 1033 \\
7 & 594.1 & -183 & 285.9 \\
8 & 11.47 & -618.5 & 1054 \\
\hline
\end{tabular}

\subsection{SIMO TOW System with IM/DD L-PPM}

Considering (19) for the $M$ copies of the optical signal with L-PPM and OC, the ABER of the total SIMO TOW system can be expressed as

$$
P_{e, a v, M}^{L-P P M}=\int_{\mathrm{h}} f_{\mathrm{h}}(\mathrm{h}) Q\left[\sqrt{\frac{L \log _{2} L}{4 M N_{0}} \sum_{m=1}^{M}\left(\eta_{m} h_{m}\right)^{2}}\right] d \mathrm{~h},
$$

Next, by using the accurate enough approximation of [65] for the Q-function, $Q(x) \approx \frac{e^{-x^{2} / 2}+3 e^{-2 x^{2} / 3}}{12}$, into (28) the latter gives

$$
\begin{aligned}
P_{e, a v, M}^{L-P P M} & \approx \frac{L}{24} \prod_{m=1}^{M} \int_{0}^{\infty} \exp \left(-\frac{\eta_{m}^{2} h_{m}^{2} L \log _{2} L}{8 M N_{0}}\right) f_{h_{m}}\left(h_{m}\right) d h_{m} \\
& +\frac{L}{8} \prod_{m=1}^{M} \int_{0}^{\infty} \exp \left(-\frac{\eta_{m}^{2} h_{m}^{2} L \log _{2} L}{6 M N_{0}}\right) f_{h_{m}}\left(h_{m}\right) d h_{m} .
\end{aligned}
$$

Thus, by using $\int_{0}^{\infty} x^{n} e^{-z x^{2}} d x=\frac{\Gamma[(n+1) / 2]}{2 z^{\frac{n+1}{2}}}, n>-1, z>0$ [59], and by substituting (3) in (29), the following ABER expression is derived for a IM/DD L-PPM SIMO TOW system with OC, under the joint impact of both skin-induced attenuation and NZB pointing errors

$$
\begin{aligned}
P_{e, a v, M}^{L-P P M} & \approx \frac{L}{24} \prod_{m=1}^{M} \frac{\psi_{m}^{2} \exp \left(-\alpha\left(\lambda_{m}\right) \delta_{m} / 2\right)^{\psi_{m}^{2}-1} \Gamma\left[\left(1-\psi_{m}^{2}\right) / 2\right]}{2\left(\eta_{m}^{2} L \log _{2} L / 8 M N_{0}\right)^{\frac{1-\psi_{m}^{2}}{2}}\left(A_{0, m} g_{m}\right)^{\psi_{m}^{2}}} \\
& +\frac{L}{8} \prod_{m=1}^{M} \frac{\psi_{m}^{2} \exp \left(-\alpha\left(\lambda_{m}\right) \delta_{m} / 2\right)^{\psi_{m}^{2}-1} \Gamma\left[\left(1-\psi_{m}^{2}\right) / 2\right]}{2\left(\eta_{m}^{2} L \log _{2} L / 6 M N_{0}\right)^{\frac{1-\psi_{m}^{2}}{2}}\left(A_{0, m} g_{m}\right)^{\psi_{m}^{2}}} .
\end{aligned}
$$

Additionally, by substituting (12) into (30) we obtain the latter expression as a function of the average electrical SNR, $\mu_{m}$

$$
\begin{aligned}
P_{e, a v, M}^{L-P P M} & \approx \frac{1}{12} \prod_{m=1}^{M} \frac{\psi_{m}^{3-\psi_{m}^{2}}\left(A_{0, m} g_{m}\right)^{1-2 \psi_{m}^{2}}\left(8 \widetilde{P}_{S} M\right)^{\frac{1-\psi_{m}^{2}}{2}} \Gamma\left[\left(1-\psi_{m}^{2}\right) / 2\right]}{2\left[\mu_{m}\left(\psi_{m}^{2}+2\right) L \log _{2} L\right]^{\frac{1-\psi_{m}^{2}}{2}}} \\
& +\frac{1}{4} \prod_{m=1}^{M} \frac{\psi_{m}^{3-\psi_{m}^{2}}\left(A_{0, m} g_{m}\right)^{1-2 \psi_{m}^{2}}\left(6 \widetilde{P}_{S} M\right)^{\frac{1-\psi_{m}^{2}}{2}} \Gamma\left[\left(1-\psi_{m}^{2}\right) / 2\right]}{2\left[\mu_{m}\left(\psi_{m}^{2}+2\right) L \log _{2} L\right]^{\frac{1-\psi_{m}^{2}}{2}}} .
\end{aligned}
$$

\section{Numerical Results}

In this section, by using the closed-form expressions derived above (18), (21), (26), (27), (30), (31), we present, over a wide range of average electrical SNR, performance results by means of the 
ABER metric for both SISO and SIMO TOW configurations than may employ either OOK or L-PPM modulation formats. In all investigated cases, the joint impact of both skin-induced attenuation and pointing errors effects are considered. Note that without loss of generality we focus on spatial diversity, i.e., the examined TOW system may support SIMO configurations where the transmitter simultaneously emits the same part of information to more than one spatially separated receiver apertures. Thus, each detector receives independent information-bearing optical signals. Specifically, when multiple receivers are assumed, i.e., $M>1$, the NZB component is also taken into account. In fact the examined system may employ from $M=1$ to $M=3$ TOW links of a typical skin thickness of $\delta=8 \mathrm{~mm}$, while the operational wavelength of the system is fixed at $\lambda=850 \mathrm{~nm}$. For each receiver aperture, it is assumed that $\eta_{m}=0.8, r_{m}=0.5 \mathrm{~mm}, \theta_{m}=30^{\circ}, \widetilde{P}_{S}=1 \mathrm{mw} / \mathrm{MHz}$ and $N_{0}=(1.3 \mathrm{pA} / \sqrt{\mathrm{Hz}})^{2}$. Thus, concerning pointing errors effects it holds that $w_{\delta, m} / r_{m}>6$ which indicates that approximation in (5) is valid. Additionally, for $M=1$, due to most precise positioning, the parameter $\psi_{1}$ should obtain its maximal value, i.e., $\psi_{1}=0.35$ for $\left(w_{\delta} / r, \mu_{x} / r, \mu_{y} / r, \sigma_{x} / r, \sigma_{y} / r\right)=(9.2,20,10,9,9)$ in comparison with SIMO configurations. Indeed, for $M>1$, due to the spacing between photodetectors, we assume also stronger or even stronger non-zero boresight pointing errors effects, i.e., $\psi_{2}, \psi_{3}=0.29$ or 0.25 , for $\left(w_{\delta} / r, \mu_{x} / r, \mu_{y} / r, \sigma_{x} / r, \sigma_{y} / r\right)=\{(9.2,20,10,12,10)$ or $(9.2,20,10,15,10)\}$, respectively. Note that by substituting $850 \mathrm{~nm}$ for $\lambda_{\mathrm{m}}$ and the corresponding parameter values of Table 1 for $a_{i}, b_{i}$, and $c_{i}$, we obtain from (4) that for the examined cases it holds that $\alpha\left(\lambda_{\mathrm{m}}\right)=1.8$. Additionally, in all cases that we present below, the numerically evaluated results from the above derived closed form mathematical expressions, are accompanied by the corresponding ones obtained with Monte Carlo simulations with $2 \times 10^{7}$ samples.

As is the case of terrestrial FSO in [2,46] results in Figure 2 are now obtained for the examined TOW with OOK system by using the corresponding (26) and (27) expressions. Figure 2 illustrates the ABER evolution for the TOW system that employs both SISO and SIMO configurations with OOK over a wide range of average electrical SNR, along with different amounts of NZB misalignment-induced fading. Significant ABER degradations are demonstrated when NZB pointing errors are getting stronger. However, very important ABER performance improvements are depicted via the use of SIMO configurations, especially for larger number of receivers. Thus, the beneficial impact of the use of multiple receivers in terms of total TOW ABER performance is highlighted. Indeed, through diversity, for instance in space, the quality of the received signal is enhanced by achieving a lower ABER value for the same average electrical SNR value. Recall that higher average BER values translate into more erroneous bits arriving at the receiver's side, which aggravates the detection capability, and thus degrades the quality of the received signal.

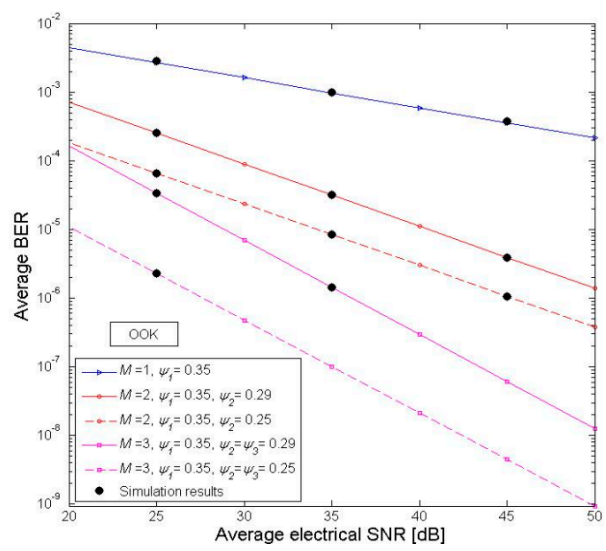

Figure 2. ABER versus electrical SNR for SISO and SIMO TOW system configurations with OOK and various NZB pointing errors conditions.

As is the case of terrestrial FSO in $[2,46]$ results in Figure 3 are now obtained for the examined TOW with 4-PPM system by using the corresponding (26) and (27), (30), and (31) expressions. Figure 3 
illustrates the corresponding to Figure 2 ABER evolution, but under the assumption that TOW employs 4-PPM modulation format instead of OOK. The ABER performance comparison between results of Figures 2 and 3 illustrates that decreased corresponding ABER values are obtained by using 4-PPM instead of OOK. Thus, it is proved that PPM modulation format can be used as an effective solution to address more drastically than OOK the ABER performance degradation of TOW links due to the joint impact of skin-induced attenuation and NZB pointing errors.

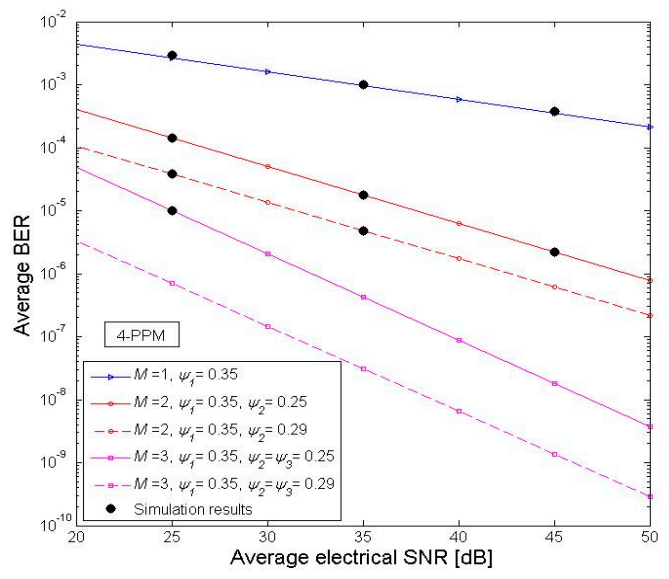

Figure 3. ABER versus electrical SNR for SISO and SIMO TOW system configurations with 4-PPM and various NZB pointing errors conditions.

As is the case of terrestrial FSO in [2,46] results in Figure 4 are now obtained for the examined TOW with 8-PPM system by using the corresponding (26) and (27), (30) and (31) expressions. Figure 4 illustrates, in turn, the corresponding to Figure 3 ABER evolution, but assuming that TOW system employs 8-PPM modulation format instead of 4-PPM. It is highlighted that TOW system outperforms when IM/DD with PPM scheme based on greater number of time slots is employed. Although greater time slots PPM ensures more power efficiency than lower one, as well as OOK, this happens at the expense of both an increased bandwidth and a more complex system's design. Indeed, when 4-PPM format is employed the laser is 'on' during a specific quarter of the 4-PPM bit interval (slot) and 'off' during the rest bit intervals (slots). Thus, the detection is a more complex procedure than that for OOK, while also the lower power consumption at the expense of the increased bandwidth requirement stems from the fact that a 4-PPM symbol consists of a pulse of constant power where information occupies one slot duration within four possible time slots with the rest of the slots being empty.

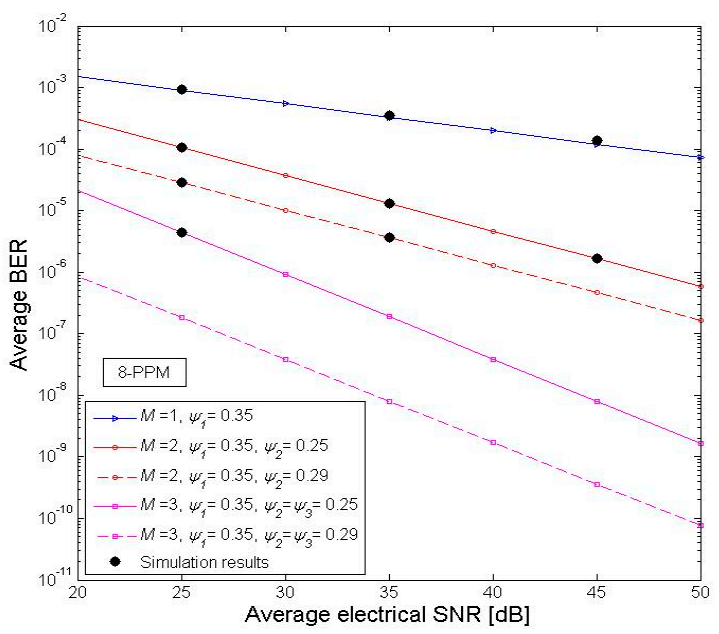

Figure 4. ABER versus electrical SNR for SISO and SIMO TOW system configurations with 8-PPM and various NZB pointing errors conditions. 


\section{Conclusions}

In this work, it has been investigated the joint impact of skin-induced attenuation along with NZB pointing errors, on the outage performance of TOW communication system with or without receivers diversity-i.e., SISO or SIMO TOW links-using IM/DD with either OOK or L-PPM formats and OC method at the receiver(s) side. Under these circumstances novel closed-form expressions for the ABER of the TOW system have been derived. Using extracted expressions, we presented proper numerical results, validated by simulations, which reveal that the combined use of receivers' diversity along with L-PPM modulation formats can significantly improve the ABER performance of typical TOW links. In this context, greater ABER performance enhancements can be obtained by increasing both the number of apertures at the receiver along with the complexity of L-PPM modulation formats. Thus, the proposed analysis is a useful tool to outbalance the major factors that have a negative impact on TOW development and design more effective TOW systems of higher availability and ABER performance.

Author Contributions: Conceptualization, G.K.V., H.E.N., and K.A.; Methodology, G.K.V., H.E.N., and K.A.; Software, G.K.V.; Validation, G.K.V., H.E.N., K.A., F.J., and K.K.M.R.; Investigation, G.K.V., H.E.N., K.A., F.J., and K.K.M.R.; Resources, G.K.V., H.E.N., K.A., F.J., and K.K.M.R.; Writing-original draft preparation, G.K.V.; Writing一review and editing, G.K.V. and H.E.N.; Supervision, G.K.V., H.E.N., and K.A.; Funding acquisition, H.E.N.

Funding: H.E.N. acknowledges the funding from the European Union's Horizon 2020 research and innovation program under grant agreement no. 777596. G.K.V., K.A., F.J., and K.K.M.R. acknowledge that this project has partially received funding from the Ajman University under grant agreement IDG-2018-A-EN-01.

Conflicts of Interest: The authors declare no conflict of interest.

\section{References}

1. Abualhoul, M.Y.; Svenmarker, P.; Wang, Q.; Andersson, J.Y.; Johansson, A.J. Free space optical link for biomedical applications. In Proceedings of the 2012 Annual International Conference of the IEEE Engineering in Medicine and Biology Society, San Diego, CA, USA, 28 August-1 September 2012; IEEE: Piscataway, NJ, USA, 2012; pp. 1667-1670.

2. Varotsos, G.K.; Nistazakis, H.E.; Petkovic, M.I.; Djordjevic, G.T.; Tombras, G.S. SIMO Optical Wireless Links with Nonzero Boresight Pointing Errors over M modeled Turbulence Channels. Elsevier Opt. Commun. 2017, 403, 391-400. [CrossRef]

3. Varotsos, G.K.; Stassinakis, A.N.; Nistazakis, H.E.; Tsigopoulos, A.D.; Peppas, K.P.; Aidinis, C.J.; Tombras, G.S. Probability of fade estimation for FSO links with time dispersion and turbulence modeled with the gamma-gamma or the IK distribution. Opt. Int. J. Light Electron Opt. 2014, 125, 7191-7197. [CrossRef]

4. Varotsos, G.K.; Nistazakis, H.E.; Volos, C.K.; Tombras, G.S. FSO links with diversity pointing errors and temporal broadening of the pulses over weak to strong atmospheric turbulence channels. Opt. Int. J. Light Electron Opt. 2016, 127, 3402-3409. [CrossRef]

5. Henniger, H.; Wilfert, O. An Introduction to Free Space Optical Communications. Radioengineering 2010, 19, 203-212.

6. Majumdar, A.K.; Arun, K. Advanced Free Space Optics (FSO); Springer: Berlin/Heidelberg, Germany, 2015; p. 397.

7. Farid, A.A.; Hranilovic, S. Outage capacity optimization for free space optical links with pointing errors. IEEE/OSA J. Lightwave Technol. 2007, 25, 1702-1710. [CrossRef]

8. Hogan, H. Data Demands: Drive Free-Space Optics. Photonics Spectra 2013, 47, 38-41.

9. Ghassemlooy, Z.; Arnon, S.; Uysal, M.; Xu, Z.; Cheng, J. Emerging optical wireless communications-advances and challenges. IEEE J. Sel. Areas Commun. 2015, 33, 1738-1749. [CrossRef]

10. Awan, M.S.; Csurgai-Horváth, L.; Muhammad, S.S.; Leitgeb, E.; Nadeem, F.; Khan, M.S. Characterization of Fog and Snow Attenuations for Free-Space Optical Propagation. JCM 2009, 4, 533-545. [CrossRef]

11. Kedar, D.; Arnon, S. Urban Optical Wireless Communication Networks: The Main Challenges and Possible Solutions. IEEE Commun. Mag. 2004, 42, S2-S7. [CrossRef] 
12. Khalighi, M.A.; Uysal, M. Survey on free space optical communication: A communication theory perspective. IEEE Commun. Surv. Tutor. 2014, 16, 2231-2258. [CrossRef]

13. Nistazakis, H.E.; Katsis, A.; Tombras, G.S. On the Reliability and Performance of FSO and Hybrid FSO Communication Systems over Turbulent Channels. In Turbulence: Theory, Types and Simulation; Series Physics Research and Technology; Marcuso, R.J., Ed.; Nova Publishers: Hauppauge, NY, USA, 2012; ISBN 978-1-61761-735-5.

14. Gil, Y.; Rotter, N.; Arnon, S. Feasibility of retroreflective transdermal optical wireless communication. Appl. Opt. 2012, 51, 4232-4239. [CrossRef] [PubMed]

15. Chapin, J.K.; Moxon, K.A.; Markowitz, R.S.; Nicolelis, M.A. Real-time control of a robot arm using simultaneously recorded neurons in the motor cortex. Nat. Neurosci. 1999, 2, 664-670. [CrossRef] [PubMed]

16. Serruya, M.D.; Hatsopoulos, N.G.; Paninski, L.; Fellows, M.R.; Donoghue, J.P. Brain-machine interface: Instant neural control of a movement signal. Nature 2002, 416, 141-142. [CrossRef] [PubMed]

17. Smith, B.; Tang, Z.; Johnson, M.W.; Pourmehdi, S.; Gazdik, M.M.; Buckett, J.R.; Peckham, P.H. An externally powered, multichannel, implantable stimulator-telemeter for control of paralyzed muscle. IEEE Trans. Biomed. Eng. 1998, 45, 463-475. [CrossRef] [PubMed]

18. Gheewala, T.R.; Melen, R.D.; White, R.L. A CMOS implantable multielectrode auditory stimulator for the deaf. IEEE J. Solid State Circuits 1975, 10, 472-479. [CrossRef]

19. Liu, T.; Bihr, U.; Anis, S.M.; Ortmanns, M. Optical transcutaneous link for low power, high data rate telemetry. In Proceedings of the 2012 Annual International Conference of the IEEE Engineering in Medicine and Biology Society (EMBC), San Diego, CA, USA, 28 August-1 September 2012; IEEE: Piscataway, NJ, USA, 2012; pp. 3535-3538.

20. Ghovanloo, M.; Najafi, K. A wideband frequency-shift keying wireless link for inductively powered biomedical implants. IEEE Trans. Circuits Syst. I Regul. Pap. 2004, 51, 2374-2383. [CrossRef]

21. Mohseni, P.; Najafi, K.; Eliades, S.J.; Wang, X. Wireless multichannel biopotential recording using an integrated FM telemetry circuit. IEEE Trans. Neural Syst. Rehabil. Eng. 2005, 13, 263-271. [CrossRef]

22. Neihart, N.M.; Harrison, R.R. A low-power FM transmitter for use in neural recording applications. In Proceeding of the 26th Annual International Conference of the IEEE Engineering in Medicine and Biology Society, San Francisco, CA, USA, 1-5 September 2004; IEEE: Piscataway, NJ, USA, 2004; Volume 1, pp. 2117-2120.

23. Miranda, H.; Gilja, V.; Chestek, C.A.; Shenoy, K.V.; Meng, T.H. HermesD: A high-rate long-range wireless transmission system for simultaneous multichannel neural recording applications. IEEE Trans. Biomed. Circuits Syst. 2010, 4, 181-191. [CrossRef] [PubMed]

24. Charles, C.T. Wireless data links for biomedical implants: Current research and future directions. In Proceedings of the Biomedical Circuits and Systems Conference BIOCAS 2007, Montreal, QC, Canada, 27-30 November 2007; IEEE: Piscataway, NJ, USA, 2007; pp. 13-16.

25. Chae, M.; Liu, W.; Yang, Z.; Chen, T.; Kim, J.; Sivaprakasam, M.; Yuce, M. A 128-channel 6mw wireless neural recording ic with on-the-fly spike sorting and uwb tansmitter. In Proceeding of the Solid-State Circuits Conference, San Francisco, CA, USA, 3-7 February 2008; IEEE: Piscataway, NJ, USA, 2018.

26. Schwartz, A.B. Cortical neural prosthetics. Annu. Rev. Neurosci. 2004, 27, 487-507. [CrossRef]

27. Hochberg, L.R.; Serruya, M.D.; Friehs, G.M.; Mukand, J.A.; Saleh, M.; Caplan, A.H.; Branner, A.; Chen, D.; Penn, R.D.; Donoghue, J.P. Neuronal ensemble control of prosthetic devices by a human with tetraplegia. Nature 2006, 442, 164. [CrossRef]

28. Abita, J.L.; Schneider, W. Transdermal optical Communications; John Hopkins APL Tech: Laurel, MD, USA, 2004; Volume 25, pp. 261-268.

29. Liu, T.; Bihr, U.; Becker, J.; Anders, J.; Ortmanns, M. In vivo verification of a $100 \mathrm{Mbps}$ transcutaneous optical telemetric link. In Proceedings of the Biomedical Circuits and Systems Conference (BioCAS), Lausanne, Switzerland, 22-24 October 2014; IEEE: Piscataway, NJ, USA, 2014; pp. 580-583.

30. Ritter, R.; Handwerker, J.; Liu, T.; Ortmanns, M. Telemetry for implantable medical devices: Part 1-Media properties and standards. IEEE Solid State Circuits Mag. 2014, 6, 47-51. [CrossRef]

31. Liu, T.; Anders, J.; Ortmanns, M. System level model for transcutaneous optical telemetric link. In Proceedings of the 2013 IEEE International Symposium on Circuits and Systems (ISCAS), Beijing, China, 19-23 May 2013; IEEE: Piscataway, NJ, USA, 2013; pp. 865-868.

32. Ackermann, D.M.; Smith, B.; Kilgore, K.L.; Peckham, P.H. Design of a high speed transcutaneous optical telemetry link. In Proceedings of the 2006 International Conference of the IEEE Engineering in Medicine 
and Biology Society, New York, NY, USA, 30 August-3 September 2006; IEEE: Piscataway, NJ, USA, 2006; pp. 2932-2935.

33. Ackermann, D.M.; Smith, B.; Wang, X.F.; Kilgore, K.L.; Peckham, P.H. Designing the optical interface of a transcutaneous optical telemetry link. IEEE Trans. Biomed. Eng. 2008, 55, 1365-1373. [CrossRef] [PubMed]

34. Yoon, Y.H.; Jung, E.S.; Song, B.S.; Lee, S.H.; Cho, J.H. Design of a transcutaneous infrared remote control for the totally implantable middle ear system. IEICE Trans. Electron. 2005, 88, 1896-1899. [CrossRef]

35. Parmentier, S.; Fontaine, R.; Roy, Y. Laser diode used in $16 \mathrm{Mb} / \mathrm{s}, 10 \mathrm{~mW}$ optical transcutaneous telemetry system. In Proceedings of the Biomedical Circuits and Systems Conference, BioCAS, Baltimore, MD, USA, 20-22 November 2008; IEEE: Piscataway, NJ, USA, 2008; pp. 377-380.

36. Trevlakis, S.E.; Boulogeorgos, A.A.A.; Karagiannidis, G.K. On the impact of misalignment fading in transdermal optical wireless communications. In Proceedings of the 7th International Conference on Modern Circuits and Systems Technologies (MOCAST), Thessaloniki, Greece, 7-9 May 2018; IEEE: Piscataway, NJ, USA, 2018; pp. 1-4.

37. Trevlakis, S.E.; Boulogeorgos, A.A.A.; Karagiannidis, G.K. Outage Performance of Transdermal Optical Wireless Links in the Presence of Pointing Errors. In Proceedings of the 2018 IEEE 19th International Workshop on Signal Processing Advances in Wireless Communications (SPAWC), Kalamata, Greece, 25-28 June 2018; IEEE: Piscataway, NJ, USA, 2018; pp. 1-5.

38. Trevlakis, S.; Boulogeorgos, A.A.; Karagiannidis, G. Signal Quality Assessment for Transdermal Optical Wireless Communications under Pointing Errors. Technologies 2018, 6, 109. [CrossRef]

39. Varotsos, G.K.; Nistazakis, H.E.; Tombras, G.S.; Aidinis, K.; Jaber, F.; Rahman, M. On the use of diversity in transdermal optical wireless links with nonzero boresight pointing errors for outage performance estimation. In Proceedings of the 2018 7th International Conference on Modern Circuits and Systems Technologies (MOCAST), Thessaloniki, Greece, 7-9 May 2018; IEEE: Piscataway, NJ, USA, 2019. in press.

40. Lange, S.; Xu, H.; Lang, C.; Pless, H.; Becker, J.; Tiedkte, H.J.; Hennig, E.; Ortmanns, M. An AC-powered optical receiver consuming $270 \mu \mathrm{W}$ for transcutaneous $2 \mathrm{Mb} / \mathrm{s}$ data transfer. In Proceedings of the $2011 \mathrm{IEEE}$ International Solid-State Circuits Conference, San Francisco, CA, USA, 20-24 February 2011; IEEE: Piscataway, NJ, USA, 2011; pp. 304-306.

41. Yang, F.; Cheng, J.; Tsiftsis, T.A. Free-space optical communication with nonzero boresight pointing errors. IEEE Trans. Commun. 2014, 62, 713-725. [CrossRef]

42. Boluda-Ruiz, R.; García-Zambrana, A.; Castillo-Vazquez, C.; Castillo-Vazquez, B. Novel approximation of misalignment fading modeled by Beckmann distribution on free-space optical links. Opt. Express 2016, 24, 22635-22649. [CrossRef] [PubMed]

43. Varotsos, G.K.; Nistazakis, H.E.; Tombras, G.S. OFDM RoFSO Links with Relays Over Turbulence Channels and Nonzero Boresight Pointing Errors. J. Commun. 2017, 12, 644-658. [CrossRef]

44. Varotsos, G.K.; Nistazakis, H.E.; Gappmair, W.; Sandalidis, H.G.; Tombras, G.S. DF Relayed Subcarrier FSO Links over Malaga Turbulence Channels with Phase Noise and Non-Zero Boresight Pointing Errors. Appl. Sci. 2018, 8, 2076-3417. [CrossRef]

45. Varotsos, G.K.; Nistazakis, H.E.; Stassinakis, A.N.; Volos, C.K.; Christofilakis, V.; Tombras, G.S. Mixed Topology of DF Relayed Terrestrial Optical Wireless Links with Generalized Pointing Errors over Turbulence Channels. Technologies 2018, 6, 121. [CrossRef]

46. Varotsos, G.K.; Nistazakis, H.E.; Gappmair, W.; Sandalidis, H.G.; Tombras, G.S. SIMO subcarrier PSK FSO links with phase noise and non-zero boresight pointing errors over turbulence channels. IET Commun. 2019, 3, 831-836. [CrossRef]

47. Navidpour, S.M.; Uysal, M.; Kavehrad, M. BER performance of free-space optical transmission with spatial diversity. IEEE Trans. Wirel. Commun. 2007, 6, 2813-2819. [CrossRef]

48. Xu, F.; Khalighi, A.; Caussé, P.; Bourennane, S. Channel coding and time-diversity for optical wireless links. Opt. Express 2009, 17, 872-887. [CrossRef] [PubMed]

49. Rachmani, R.; Arnon, S. Wavelength diversity in turbulence channels for sensor networks. In Proceedings of the 2010 IEEE 26th Convention of Electrical and Electronics Engineers in Israel (IEEEI), Eliat, Israel, 17-20 Novmber 2010; IEEE: Piscataway, NJ, USA, 2010; pp. 915-918.

50. Tsiftsis, T.A.; Sandalidis, H.G.; Karagiannidis, G.K.; Uysal, M. Optical wireless links with spatial diversity over strong atmospheric turbulence channels. IEEE Trans. Wirel. Commun. 2009, 8, 951-957. [CrossRef] 
51. Nistazakis, H.E.; Tombras, G.S. On the use of wavelength and time diversity in optical wireless communication systems over gamma-gamma turbulence channels. Opt. Laser Technol. 2012, 44, 2088-2094. [CrossRef]

52. Nistazakis, H.E. A time-diversity scheme for wireless optical links over exponentially modeled turbulence channels. Opt. Int. J. Light Electron Opt. 2013, 124, 1386-1391. [CrossRef]

53. Garcia-Zambrana, A.; Boluda-Ruiz, R.; Castillo-Vazquez, C.; Castillo-Vazquez, B. Transmit alternate laser selection with time diversity for FSO communications. Opt. Express 2014, 22, 23861-23874. [CrossRef] [PubMed]

54. Prabu, K.; Cheepalli, S.; Kumar, D.S. Analysis of PolSK based FSO system using wavelength and time diversity over strong atmospheric turbulence with pointing errors. Opt. Commun. 2014, 324, 318-323. [CrossRef]

55. Gappmair, W.; Hranilovic, S.; Leitgeb, E. Performance of PPM on terrestrial FSO links with turbulence and pointing errors. IEEE Commun. Lett. 2010, 14, 468-470. [CrossRef]

56. Manea, V.; Dragomir, R.; Puscoci, S. OOK and PPM modulations effects on bit error rate in terrestrial laser transmissions. Telecomunicat II Anul. LIV 2011, 2, 55-61.

57. Elganimi, T.Y. Performance comparison between OOK, PPM and pam modulation schemes for free space optical (FSO) communication systems: Analytical study. Int. J. Comput. Appl. 2013, 79, 22-27.

58. Boluda-Ruiz, R.; Garcia-Zambrana, A.; Castillo-Vazquez, B.; Castillo-Vazquez, C. Impact of nonzeroboresight pointing error on ergodic capacity of MIMO FSO communication systems. Opt. Express 2016, 24, 3513-3534. [CrossRef] [PubMed]

59. Gradshteyn, I.S.; Ryzhik, I.M. Table of Integrals, Series, and Products, 6th ed.; Academic: New York, NY, USA, 2000.

60. Helstrom, C.W. Probability and Stochastic Processes for Engineers; Macmillan Coll Division: London, UK, 1991.

61. Ijaz, M.; Ghassemlooy, Z.; Ansari, S.; Adebanjo, O.; Le Minh, H.; Rajbhandari, S.; Gholami, A. Experimental investigation of the performance of different modulation techniques under controlled FSO turbulence channel. In Proceedings of the 5th International Symposium on Telecommunications (IST2010), Tehran, Iran, 4-6 December 2010; IEEE: Piscataway, NJ, USA, 2010; pp. 59-64.

62. Al-Habash, M.A.; Andrews, L.C.; Phillips, R.L. Mathematical model for the irradiance probability density function of a laser beam propagating through turbulent media. Opt. Eng. 2001, 40, 1554-1562. [CrossRef]

63. The Wolfarm Functions Site. 2008. Available online: http:/functions.wolfarm.com (accessed on 22 May 2019).

64. Audeh, M.D.; Kahn, J.M. Performance evaluation of L-pulse-position modulation on non-directed indoor infrared channels. In Proceedings of the IEEE International Conference on Communications, ICC'94, SUPERCOMM/ICC'94, Conference Record, Serving Humanity Through Communications, New Orleans, LA, USA, 1-5 May 1994; IEEE: Piscataway, NJ, USA, 1994.

65. Chiani, M.; Dardari, D.; Simon, M.K. New exponential bounds and approximations for the computation of error probability in fading channels. Trans. Wirel. Commun. 2003, 2, 840-845. [CrossRef]

66. Alouini, M.S.; Simon, M.K. An MGF-based performance analysis of generalized selection combining over Rayleigh fading channels. IEEE Trans. Commun. 2000, 48, 401-415. [CrossRef]

(C) 2019 by the authors. Licensee MDPI, Basel, Switzerland. This article is an open access article distributed under the terms and conditions of the Creative Commons Attribution (CC BY) license (http://creativecommons.org/licenses/by/4.0/). 